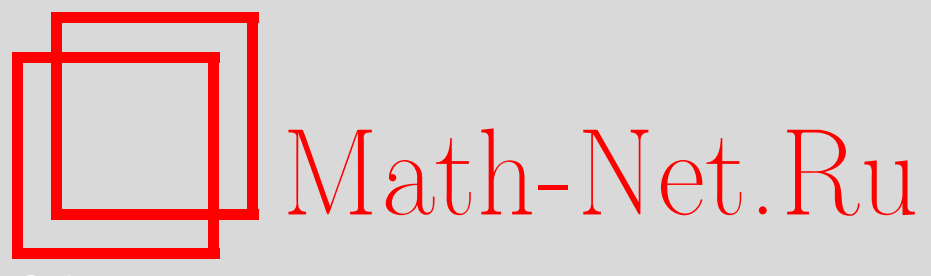

В. И. Арнольд, Насколько случайны арифметические прогрессии дробных долей?, УМН, 2008, том 63, выпуск $2,5-20$

DOI: https://doi.org/10.4213/rm9191

Использование Общероссийского математического портала Math-Net.Ru подразумевает, что вы прочитали и согласны с пользовательским соглашением http://www . mathnet.ru/rus/agreement

Параметры загрузки:

IP : 54.162 .85 .209

26 апреля 2023 г., 08:05:32

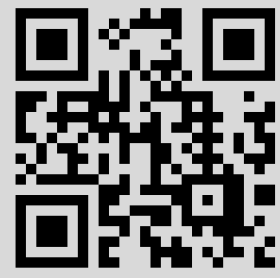


УДК $511+519.218 .84$

\title{
Насколько случайны арифметические прогрессии дробных долей?
}

\author{
В. И. Арнольд
}

Для остатков от деления на вещественное число $N$ членов арифметической прогрессии, шаг которой соизмерим с $N$, доказывается стремление к 0 параметра стохастичности Колмогорова $\lambda_{n}$ последовательности остатков при стремлении к бесконечности числа $n$ членов последовательности.

Напротив, для случая несоизмеримого с $N$ шага прогрессии приведены примеры, когда параметр стохастичности $\lambda_{n}$ не только не стремится к нулю при стремлении $n$ к бесконечности, но принимает (изредка) сколь угодно большие значения.

Как слишком малые, так и слишком большие значения параметра стохастичности делают гипотезу о случайности последовательности маловероятной, так что длинные арифметические прогрессии дробных долей, по-видимому, гораздо менее стохастичны, чем геометрические (доставляющие параметру стохастичности умеренные значения, подобно истинно случайным последовательностям).

Библиография: 5 названий.

\section{1. Измерение степени случайности}

В 1933 году А. Н. Колмогоров [1] открыл замечательное статистическое свойство последовательностей независимых наблюдений любой вещественной случайной величины (имеющей непрерывную функцию распределения).

Введенный им параметр стохастичности $\lambda_{n}$ позволяет судить, насколько правдоподобно предположение, что заданные $n$ наблюденных чисел представляют собой независимые значения одной и той же случайной величины.

Параметр стохастичности Колмогорова $\lambda_{n}$ выражается им через эмпирическую считающую функиию $C_{n}$, описывающую наблюденные значения

$$
x_{1} \leqslant x_{2} \leqslant \cdots \leqslant x_{n}
$$

(записанные здесь в порядке возрастания значений).

Значение считающей функции $C_{n}$ в точке $X \in \mathbb{R}$ есть число $C_{n}(X)$ не превосходящих $X$ наблюденных значений.

Работа выполнена при поддержке РФФИ (грант № 05-01-00104).

(С) В.И. Арнольд, 2008 
Иначе говоря,

$$
C_{n}(X)= \begin{cases}0 & \text { при } X<x_{1}, \\ m & \text { при } x_{m} \leqslant X<x_{m+1}, \\ n & \text { при } X \geqslant x_{n} .\end{cases}
$$

Колмогоров сравнивает эту эмпирическую считающую функцию $C_{n}$, описывающую $n$ наблюденных значений, с "теоретической считающей функuией" $C_{0}$, значения которой определяются так:

$$
\begin{aligned}
C_{0}(X)= & (\text { математическое ожидание числа } \\
& \text { не превосходящих } X \text { значений из } n) \\
= & (n \cdot(\text { вероятность события } x \leqslant X)) .
\end{aligned}
$$

Далее Колмогоров рассматривает отклонение эмпирической считающей функции от теоретической, оценивая его в равномерной метрике:

$$
F_{n}=\sup _{X}\left|C_{n}(X)-C_{0}(X)\right|
$$

(в отличие от своего предшественника Мизеса, использовавшего среднее квадратичное отклонение).

Чтобы получить параметр стохастичности $\lambda_{n}$, Колмогоров нормирует величину отклонения, деля ее на $\sqrt{n}$ (т. е. на величину ожидаемого для $n$ независимых наблюдений случайной величины отклонения)

$$
\lambda_{n}=\frac{F_{n}}{\sqrt{n}} .
$$

Этот параметр стохастичности Колмогорова $\lambda_{n}$ набора $n$ наблюденных значений $\left\{x_{1}, \ldots, x_{n}\right\}$ сам является случайной величиной. Удивительная теорема Колмогорова [1] описывает асимптотику распределения этой случайной величины $\lambda_{n}$ при $n \rightarrow \infty$.

Оказывается, эта случайная величина $\lambda_{n}$ распределена (в пределе при $n \rightarrow \infty$, с равномерной сходимостью функций распределения к предельному значению Колмогорова $\Phi)$ универсальным стандартным образом, не зависящим от распределения исходной величины $x$.

А именно, Колмогоров доказал, что предельное распределение параметра стохастичности (на оси вещественной переменной $\Lambda$ ) таково: если

$$
\Phi(\Lambda)=(\text { вероятность события } \lambda \leqslant \Lambda),
$$

то $\Phi(\Lambda)$ есть (при всяком $\Lambda>0$ ) сумма ряда

$$
\Phi(\Lambda)=\sum_{k \in \mathbb{Z}}(-1)^{k} \mathrm{e}^{-2 k^{2} \Lambda^{2}} .
$$

ПримеР. Приближенные значения этой (монотонной) функции распределения Колмогорова $\Phi$ таковы: ${ }^{1}$

$$
\Phi(0.2) \approx 0.0000, \quad \Phi(2.2) \approx 1.0000,
$$

\footnotetext{
${ }^{1}$ Мы обозначаем далее через 0.1 числа $1 / 10$ и т. п.
} 
а для промежуточных значений параметра стохастичности $\Lambda$ Колмогоров указывает следующие приближенные значения функции $\Phi$ (с четырьмя знаками):

\begin{tabular}{|c||c|c|c|c|c|c|c|c|c|}
\hline$\Lambda$ & 0.4 & 0.6 & 0.8 & 1.0 & 1.2 & 1.4 & 1.6 & 1.8 & 2.0 \\
\hline $10^{4} \Phi$ & 0028 & 1357 & 4558 & 7300 & 8877 & 9603 & 9888 & 9969 & 9993 \\
\hline
\end{tabular}

В этом распределении Колмогорова как слишком малые (скажем, меньшие, чем 0.4), так и слишком большие (скажем, превосходящие 1.8) значения параметра стохастичности $\Lambda$ маловероятны (имеют вероятность меньше одной трети процента).

Поэтому наблюденное значение $\lambda_{n}$ позволяет судить о случайности наблюденной последовательности $n$ значений: эта случайность маловероятна, если наблюденное значение $\lambda_{n}$ слишком мало или слишком велико.

Среднее (по распределению Колмогорова $\Phi$ ) значение параметра стохастичности $\Lambda$ составляет $\Lambda=\sqrt{\pi / 2} \ln 2 \approx 0.869$. Медианное значение (для которого и бо́льшие, и ме́ньшие значения параметра стохастичности имеют в распределении Колмогорова $\Phi$ вероятность $1 / 2$ ) составляет $\Lambda \approx 0.83$.

В настоящей работе я применил этот метод к исследованию “степени случайности" арифметических прогрессий остатков от деления на какое-либо число $N$.

ПримеР. Последовательность $n$ двузначных чисел

$$
37,74,11,48,85,22,59,96,33,70,7,44, \ldots
$$

(членами которой являются остатки от деления на $N=100$ чисел $37 x$, где $x=1,2,3, \ldots, n)$ кажется на вид (псевдо)случайной.

Для объективной оценки степени случайности последовательности $n$ чисел сосчитаем значение вычисленного по ним параметра Колмогорова $\lambda_{n}$. Сравнивая полученное значение с распределением Колмогорова $\Phi$, можно истолковать маловероятные значения параметра $\lambda_{n}$ как признак неслучайности исследуемой последовательности, а вероятные значения - как подтверждение ее (псевдо)случайности.

В качестве “теоретического распределения" $C_{0}$ (для арифметических прогрессий остатков от деления на $N$ ) использовалось равномерное распределение на отрезке значений от 0 до $N$ (с линейной функцией распределения: $C_{0}(X)=\frac{n}{N} X$ при $\left.0 \leqslant X<N\right)$.

Равномерность распределения остатков от деления на $N$ членов арифметической прогрессии, разность которой несоизмерима с $N$, доказана Г. Вейлем.

Описанное выше измерение степени случайности параметром Колмогорова $\lambda_{n}$ можно применять и к другим (псевдослучайным) последовательностям.

Проведенные в работе [2] вычислительные эксперименты подтвердили в этом смысле “случайность" геометрических прогрессий остатков

$$
\left\{a^{x}(\bmod N)\right\}, \quad x=1,2, \ldots, n,
$$

а также последовательностей остатков от деления на $N$ чисел Фибоначчи (и других рекуррентных последовательностей экспоненциального роста). 
“Теоретическое распределение" $C_{0}$ и здесь предполагалось равномерным (на отрезке от 0 до $N$ ), хотя равномерность распределения дробных долей геометрических прогрессий общего вида и не доказана (“адиабатические" доводы в пользу справедливости этой давней гипотезы о равномерном распределении дробных долей геометрических прогрессий обсуждаются в книге [3]).

Вычисленные в этих примерах значения показателя стохастичности Колмогорова получались обычно порядка $\lambda_{n} \approx 0.7$, или 0.8 , или 0.9 . В этом смысле дробные доли геометрических прогрессий ведут себя как независимые наблюдения равномерно распределенной (на отрезке от 0 до 1) случайной величины.

Напротив, для арифметических прогрессий целочисленных остатков и для последовательностей остатков от деления на фиксированное число $N$ последовательных $n$ простых чисел получались столь малые значения параметра стохастичности Колмогорова $\lambda_{n}$, что вероятность такой его величины для истинно случайных последовательностей составляет малые доли процента.

В случае простых чисел в качестве "теоретического распределения" $C_{0}$ использовалось распределение Лежандра-Чебышёва, чьей функцией распределения является интегральный логарифм.

Логарифм большого числа медленно меняется с этим числом, поэтому последовательность (больших) простых чисел ведет себя скорее как арифметическая прогрессия (с шагом, лишь слегка увеличивающимся вдоль последовательности).

Одним из наиболее известных применений параметра стохастичности Колмогорова был его анализ [4] статистических данных учеников Т. Д. Лысенко, стремившихся опровергнуть генетическую статистику Менделя, подсчитывая эмпирические отклонения от нее в нескольких тысячах экспериментов.

Анализ Колмогорова показал, что эти данные не опровергают закон Менделя, а подтверждают его, а именно, если бы импирически наблюденные отклонения оказались меньшими, чем в описанных экспериментах, то это свидетельствовало бы о том, что данные фальсифицированы, в то время как опубликованные учениками Лысенко наблюдения доставляют как раз обычные значения параметра стохастичности Колмогорова $\lambda_{n}$ (не далекое от его среднего по распределению Колмогорова значения, составляющего примерно 0.87).

В настоящей работе подробно исследуются арифметические прогрессии остатков

$$
\{u x(\bmod v)\}, \quad x=1,2, \ldots, n,
$$

с целыми $u$ и $v$.

Доказано, что в этом случае значения параметра стохастичности Колмогорова $\lambda_{n}$ стремятся $\kappa$ нулю при $n \rightarrow \infty$, так что “степень случайности” такой арифметической прогрессии остатков уменьшается до нуля с ростом длины последовательности.

Выбором масштаба измерения эта задача сводится к исследованию арифметических прогрессий дробных долей,

$$
\{k x(\bmod 1)\}, \quad x=1,2, \ldots, n,
$$

где $k=u / v$. 
Для арифметических прогрессий дробных долей с любой рачиональной разностъю $k$ получается стремящийся $\kappa$ нулю при $n \rightarrow \infty$ параметр случайности Колмогорова $\lambda_{n}$ (что указывает на асимптотическую неслучайность такой длинной прогрессии).

ЗАмечАниЕ. Параметр стохастичности Мизеса тоже стремится здесь к нулю (поскольку среднее квадратичное меньше равномерной нормы).

Напротив, в отличие от случая рациональных $k$ для иррациональных разностей $k$ в статъе приведены примеры, когда параметр Колмогорова $\lambda_{n}$ арифметической прогрессии $n$ дробных долей не стремится $\kappa 0$ при стремлении $n \kappa$ бесконечности (и даже когда он изредка неограниченно растет до сколь угодно больших, хотя и не превосходящих $\sqrt{n}$, значений, если длина последовательности $n$ достаточно велика). Для параметра стохастичности Мизеса тоже наблюдается подобный рост.

Как обстоит дело со случайностью арифметической прогрессии дробных долей шага $k$, для большинства иррациональных чисел $k$ остается неясным: все исследованные явления доказаны лишь для образующего множество меры нуль набора значений шага $k$.

Наблюденные большие значения параметра стохастичности $\lambda_{n}$ обнаружены лишь при редких специальных значениях числа $n$ членов последовательности. Быть может, средние (по длине последовательности) значения параметра стохастичности

$$
\widehat{\lambda}_{n}=\frac{\lambda_{1}+\cdots+\lambda_{n}}{n}
$$

менее хаотически зависят от арифметических свойств шага $k$ рассматриваемой арифметической прогрессии.

Но я не провел соответствующих экспериментов и не берусь предсказывать поведение чисел $\widehat{\lambda}_{n}$ для типичных вещественных значений шага $k$.

Для исследования этой зависимости "степени случайности" от параметра $k$ можно прибегнуть и к локальному усреднению по $k$ (в окрестности фиксированного значения), т. е. к рассмотрению слабых асимптотик в смысле статьи [5].

Это усреднение нужно было бы исследовать и в непрерывном случае $(k \in \mathbb{R})$, и в дискретном $\left((u, v) \in \mathbb{Z}^{2}\right)$.

При этом в непрерывном случае можно было бы проводить усреднение либо по мере Лебега на оси $k \in \mathbb{R}$, либо по инвариантной мере Гаусса-Кузьмина, обычной в теории цепных дробей (инвариантна она относительно преобразования $z \mapsto\{1 / z\}$ интервала $(0,1))$.

Хотя эти две меры (на оси значений $k$ ) и различны, можно предполагать, что "слабые асимптотики", доставляемые усреднением по той и по другой мере в окрестности одной и той же точки $k$, совпадут: "среднее поведение" чисел $\lambda_{n}$ или хотя бы чисел $\widehat{\lambda}_{n}$ будет одинаковым.

В дискретном случае речь идет об усреднении исследуемых величин $\lambda_{n}$ и $\widehat{\lambda}_{n}$ по окрестности целой точки $(m u, m v) \in \mathbb{Z}^{2}$ фиксированного (или медленно растущего с $m$ ) радиуса, и об исследовании асимптотического поведения этих средних при $m \rightarrow \infty$ (когда направление $(u: v) \in \mathbb{R P}^{1}$ фиксировано). 
Здесь можно даже предполагать сходство между слабыми асимптотиками в непрерывном и в дискретном вариантах задачи (подобно тому, как распределение Гаусса-Кузьмина элементов цепных дробей случайных вещественных чисел воспроизводится в дискретном варианте как асимптотика средних по шарам на дискретной плоскости $\mathbb{Z}^{2}$ квадратных уравнений $x^{2}+p x+q=0$, определяющих квадратичные иррациональности, для которых вычисляется усредненная статистика периодических цепных дробей).

Но я не провел даже и экспериментального исследования описанных выше усреднений для параметра Колмогорова арифметических прогрессий дробных долей, так как все мои вычислительные эксперименты проводились вручную, без компьютера.

Разумеется, теорема Колмогорова доказана им для вещественных случайных величин с непрерывной функцией распределения. Мы же будем применять его формулы к дискретному случаю величин со значениями из $\mathbb{Z}$ или из $\mathbb{Z}_{N}$, или даже из $\mathbb{Q}$.

Я предполагаю, что, хотя это и не доказано (и даже не отмечено) в [1], распределение Колмогорова дает хорошее приближение и в этом дискретном случае.

\section{2. Арифметические прогрессии дробных долей с рациональной разностью}

Здесь будет доказано стремление к нулю при $n \rightarrow \infty$ значения параметра стохастичности Колмогорова $\lambda_{n}$ набора первых $n$ членов арифметической прогрессии остатков от деления на $N$ чисел $k x(x=1,2, \ldots, n)$, когда шаг прогрессии, $k$, соизмерим с числом $N$.

Выбрав $N$ за единицу измерения на вещественной оси, мы приходим к арифметической прогрессии дробных долей $(\bmod 1)$ с рациональным шагом.

Пусть $k=u / v,(u, v)=1,-$ рациональное число (будем считать $u>0$, $v>0)$.

Рассмотрим арифметическую прогрессию дробных долей, т. е. остатков от деления на 1:

$$
r(x)=\{k x\}, \quad x=1,2, \ldots, n .
$$

[Нам будет удобно считать дробной долей целого числа единицу, так что $t=$ $[t]+\{t\}, 0<\{t\} \leqslant 1$.

Зафиксируем значение $X \in\{1,2, \ldots, n\}$.

Рассмотрим число

$$
R=\{k X\}=\frac{\rho}{v}
$$

с целым числителем, $1 \leqslant \rho \leqslant v$.

Для этого целого числа $\rho$ имеем

$$
X u=M v+\rho \quad(\text { так что } \rho=v R, \quad M=[k X]=k X-R) .
$$


На плоскости (рис. 1) с координатами $(x, m)$ рассмотрим параллелограмм (зависящий от параметров $k, n, R$ )

$$
\Pi: \quad\left\{\begin{array}{l}
0<x \leqslant n, \\
k x-R \leqslant m<k x .
\end{array}\right.
$$

Обозначим через $S(X)$ его площадь: это - рациональное число

$$
S(X)=n R=\frac{n \rho}{v} \in \mathbb{Q} .
$$

Обозначим через \# $(X)$ число лежащих в П целых точек (учитывая дважды проведенную на рисунке часть границы, но не учитывая дополнительную ее часть, где $x=0$ или $m=k x)$.

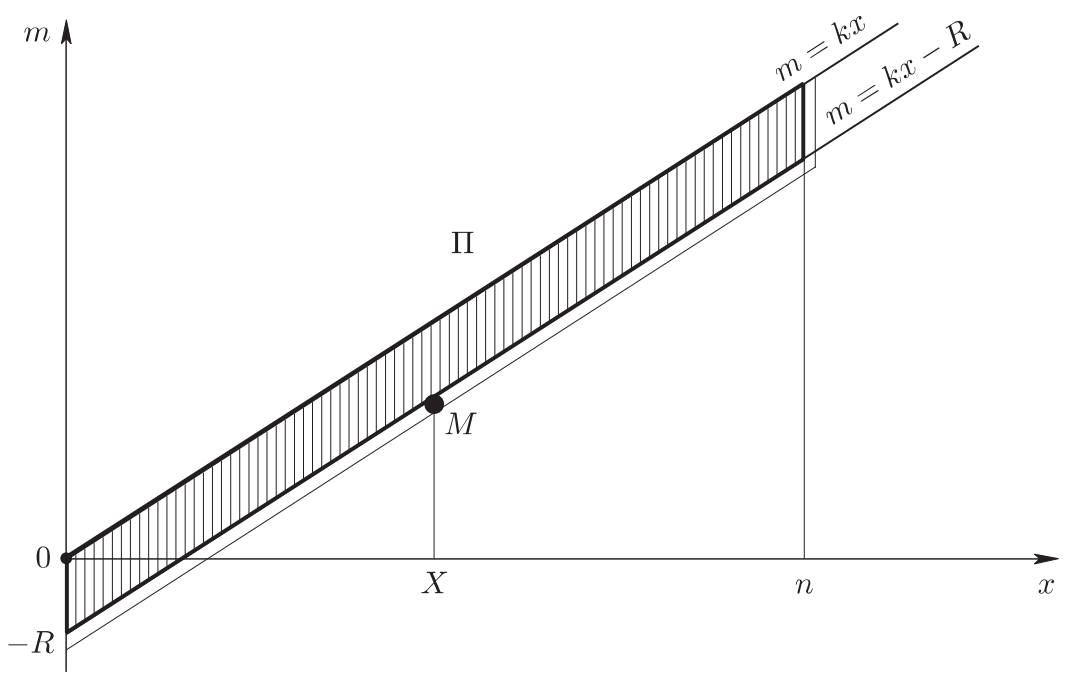

Рис. 1. Параллелограмм П (предшественников значения $R=\{k X\}$ среди первых $n$ членов арифметической прогрессии дробных долей кратных $k$ чисел)

На границе и в П лежит точка $(X, M=[k X]=k X-R)$.

Теорема 1. Если $n=c v+w$, где $0<w<v$-остаток от деления $n$ на $v$, mo

$$
|S(X)-\#(X)| \leqslant w .
$$

ПримеР. Если $n$ делится на $v$, то площадь параллелограмма П равна числу целых точек в нем:

$$
S(X)=\#(X) .
$$

СЛЕДСТВИЕ. При $n \rightarrow \infty$, параметр стохастичности Колмогорова стремится $к$ нулю:

$$
\lim _{n \rightarrow \infty} \max _{1 \leqslant X \leqslant n} \frac{|S(X)-\#(X)|}{\sqrt{n}}=0 .
$$


Ибо числитель лежит между 0 и $v$ по теореме 1 , а знаменатель стремится $\mathrm{k} \infty$.

Основой доказательства теоремы 1 является

ЛЕмма 1. При $n=v$ площадь параллелограмма П равна числу цельх точек в нем:

$$
S(X)=\#(X) .
$$

ДокАЗАТЕЛЬСтво. Рассмотрим (содержащий П) параллелограмм со стороной $R=1$, т. е.

$$
P:\left\{\begin{array}{l}
0<x \leqslant n, \\
k x-1 \leqslant m<k x .
\end{array}\right.
$$

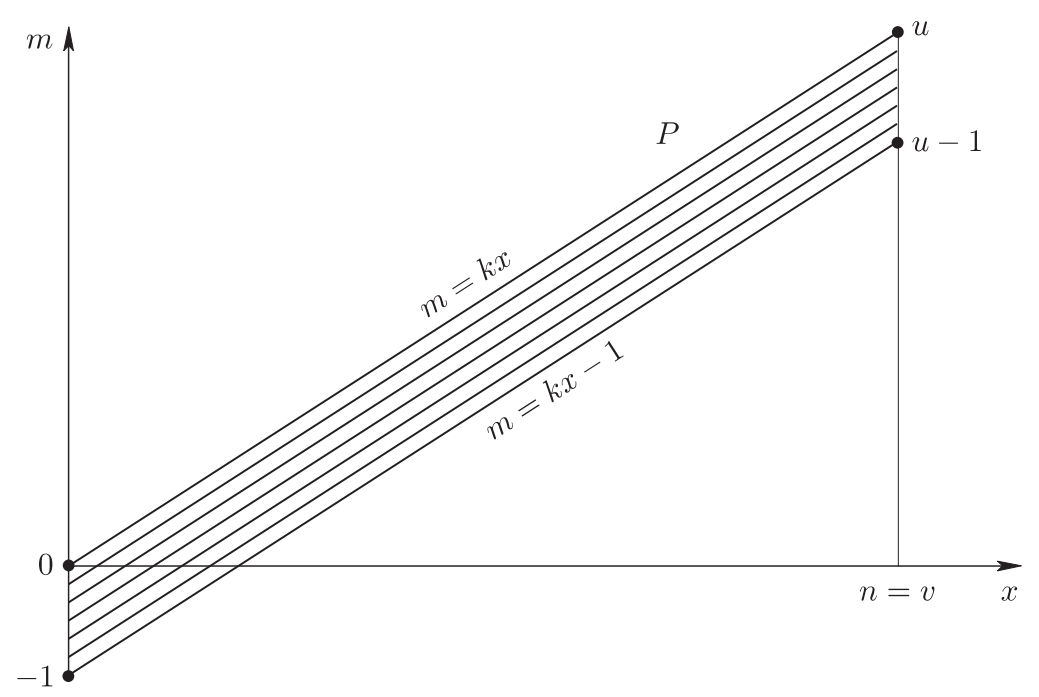

Рис. 2. Разбиение целочисленного параллелограмма $P$ на $n=v$ слоев (по одной целой точке в каждом слое)

Его площадь есть $n$. Разделим его на $v$ слоев косыми линиями (рис. 2)

$$
m=k x-\frac{\xi}{v}, \quad \xi=1, \ldots, v .
$$

Каждый слой имеет площадь $n / v(=1$ в лемме 1$)$.

В параллелограмме $P$ лежат ровно $n$ целых точек $(x, m=[k x]$ ) (по одной на каждой вертикали $x=$ const). Каждая из этих $n$ точек лежит на одной из $n$ косых прямых линий $(*)$.

При этом ни на какой из этих косых прямых не лежат две целых точки из $P$. Действительно, если бы были две точки $(x, m)$ и $\left(x^{\prime}, m^{\prime}\right)$ с общим $\xi$, то

$$
\begin{aligned}
m-k x & =m^{\prime}-k x^{\prime}, \\
v\left(m-m^{\prime}\right) & =u\left(x-x^{\prime}\right) .
\end{aligned}
$$

Но $(u, v)=1$, значит, $x-x^{\prime}=s u$, где $s-$ целое. 
Но $\left|x-x^{\prime}\right|<v$, значит, $x=x^{\prime}$, а потому $m=m^{\prime}$.

Стало быть, на каждой косой прямой $(\xi \in\{0,1, \ldots, v-1\})$ имеется ровно одна целая точка параллелограмма $P$, и других целых точек в нем нет. При этом те $\rho$ косых, для которых $0<\xi \leqslant \rho$, принадлежат параллелограмму П. Значит, параллелограмм П содержит ровно $\rho$ целых точек (а именно, точек $(x, m=[k x]=k x-\xi / v)$, где $\xi=1,2, \ldots, \rho)$. Его площадь тоже равна $\rho$. Итак, при $n=v$ имеем

$$
S(X)=\#(X)
$$

(для любой точки $X$, определяющей параллелограмм П и его площадь $\rho$ ).

Лемма 1 доказана.

ЛЕмма 2. Если $n=$ сv делится на $v$, то площадь параллелограмма П равна числу целых точек в нем:

$$
S(X)=\#(X) .
$$

ДокАЗАТЕЛьство. Разобьем параллелограмм П вертикалями $x=s v$ на $c$ кусков $\left(\Pi_{1}, \Pi_{2}, \ldots, \Pi_{c}\right)$, где в

$$
\Pi_{s}:\left\{\begin{array}{l}
(s-1) v<x \leqslant s v \\
k x-R \leqslant m<k x
\end{array}\right.
$$

где $R=\{k X\}$.

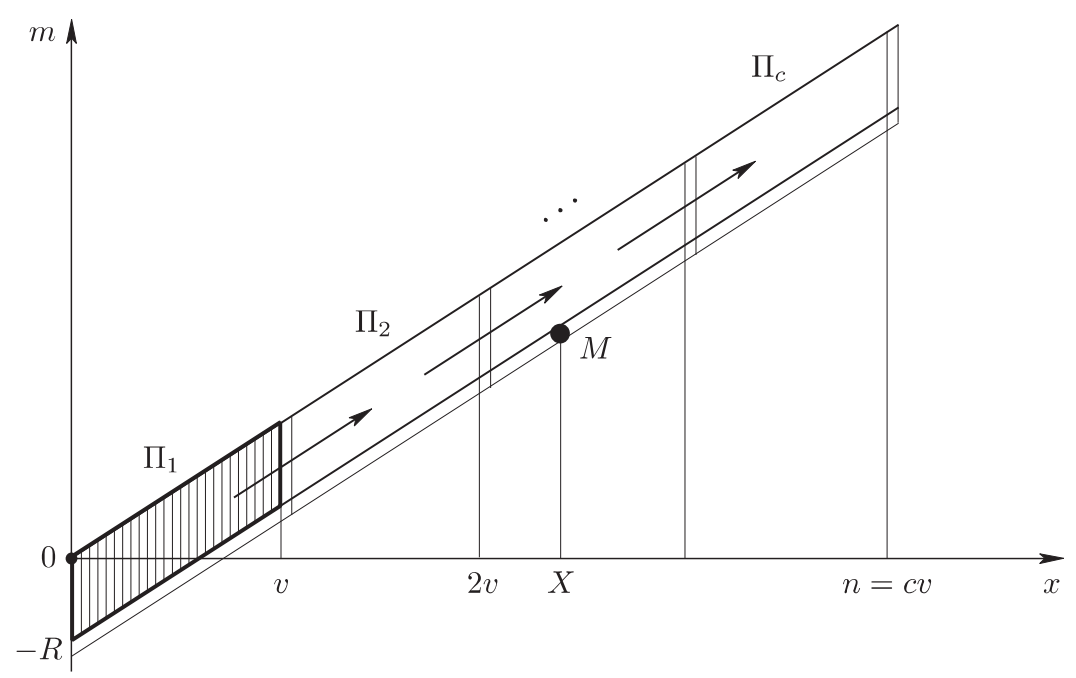

Рис. 3. Разбиение параллелограмма П высоты $c v$ на $c$ параллелограммов $\Pi_{s}$ высоты $v$

Параллелограмм $\Pi_{s}$ (рис. 3$)$ получается из $\Pi_{1}$ сдвигом на целочисленный вектор

$$
(x, m) \mapsto(x+(s-1) v, m+(s-1) u) .
$$

Поэтому в каждом из этих параллелограммов $\Pi_{s}$ одинаковое число целых точек $\rho$, и площадь каждого из них (по лемме 1 ) тоже равна $\rho$. Итак,

$$
S(X)=c \rho, \quad \#(X)=c \rho,
$$


что и доказывает лемму 2.

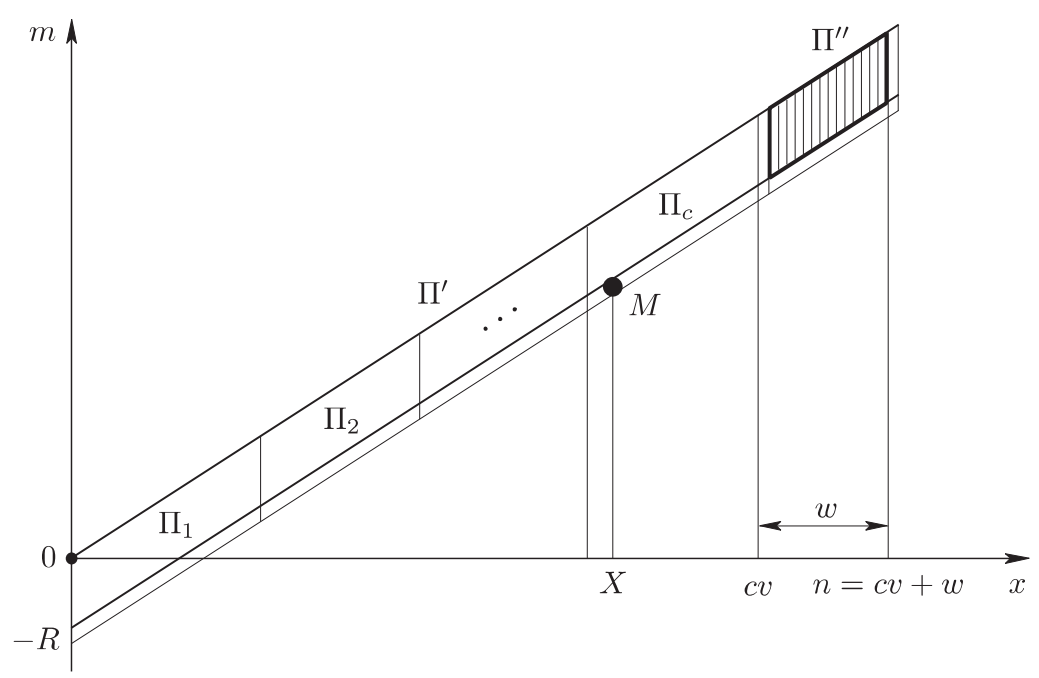

Рис. 4. Разбиение параллелограмма П высоты $c v+w$ на части $\Pi^{\prime}$ и $\Pi^{\prime \prime}$ (высот $c v$ и $w$ )

Лемма 3. Рассмотрим разбиение параллелограмма П вертикалью $x=c v$ на две части (рис. 4):

$$
\Pi^{\prime}: \quad\left\{\begin{array}{l}
0<x \leqslant c v, \\
k x-R \leqslant m<k x,
\end{array} \quad \Pi^{\prime \prime}:\left\{\begin{array}{l}
c v<x \leqslant(n=c v+w), \\
k x-R \leqslant m<k x .
\end{array}\right.\right.
$$

Тогда число чельх точек \#' и площадь $S^{\prime}$ совпадают для $\Pi^{\prime}$, а для $\Pi^{\prime \prime}$ оба эти числа не превосходят остаток $w$ от деления числа $n=c v+w$ на $v$ :

$$
\#^{\prime \prime} \leqslant w, \quad S^{\prime \prime} \leqslant w .
$$

ДоказАтельство. Первое есть лемма 2. Площадь параллелограмма П" есть $w R \leqslant w$ (так как $R \leqslant 1)$. Число целых точек в $\Pi^{\prime \prime}$ также не превосходит $w$, (так как на каждой из $w$ вертикалей $x=c v+r(r=1,2, \ldots, w)$ лежит в $\Pi^{\prime \prime}$ не более одной целой точки, поскольку $R \leqslant 1)$. Итак,

$$
0<S^{\prime \prime} \leqslant w, \quad 0<\#^{\prime \prime} \leqslant w .
$$

Из леммы 3 следует, что $\left|S^{\prime \prime}-\#^{\prime \prime}\right| \leqslant w$. А поскольку $S^{\prime}=\#^{\prime}$ по лемме 2 , то мы заключаем для

$$
S=S^{\prime}+S^{\prime \prime}, \quad \#=\#^{\prime}+\#^{\prime \prime},
$$

что

$$
|S-\#|=\left|S^{\prime \prime}-\#^{\prime \prime}\right| \leqslant w,
$$

что и доказывает теорему, а тем самым и следствие. 


\section{3. Арифметические прогрессии дробных долей с иррациональным шагом $k$}

Исследованию случая, когда шаг $k$ арифметической прогрессии дробных долей иррационален, я предпошлю некоторые свойства арифметических прогрессий дробных долей, для которых иррациональность шага $k$ возможна, но не обязательна.

Дело в том, что если бы в доказательстве предельного соотношения (1) можно было бы установить некоторую равномерность по отношению к рациональному параметру $k=u / v$ (например, если бы разность $|S-\#|$ была равномерно ограниченной сверху), то можно было бы перейти при вычислении предела (1) к иррациональным $k$ от приближающих их рациональных, для которых $\lambda_{n} \rightarrow 0$.

Следующий результат показывает, однако, что такие равномерные в $\mathbb{Q}$ оценки невозможны: пределы при $n \rightarrow \infty$ достигаются для разных рациональных значений $k$ с разной скоростью, и равномерной сходимости нет.

Теорема 2. Разности $S-\#$ площадей и чисел цельх точек параллелограммов П, построенных для всех рациональных чисел $k=u / v$ nри всех $n$ u $X$, не ограничены сверху: они достигают сколь угодно больших значений для подходящих наборов челых чисел $(u, v, n, X)$.

ДокАЗАТЕЛЬСтво. Положим $u=2 n+1, v=2 n$, так что

$$
k=\frac{2 n-1}{2 n}=\frac{1}{1+\frac{1}{2 n-1}} .
$$

Вычисляя значения площади $S(X)=R n$ и числа целых точек \# $(X)$ в соответствующих параллелограммах

$$
\Pi:\left\{\begin{array}{l}
0<x \leqslant n, \\
k x-R \leqslant m<k x, \quad \text { где } R=\{k X\},
\end{array}\right.
$$

при $X=1, \ldots, n$, мы получаем, вычислив сперва рациональное число $R=\rho / v$, следующие значения: $\rho=u X-v M, S=\rho / 2$, так что таблица значений такова:

\begin{tabular}{|c||c|c|c|c|c|}
\hline$X$ & 1 & 2 & 3 & $s$ & $n$ \\
\hline$u X$ & $2 n-1$ & $4 n-2$ & $6 n-3$ & $2 s n-s$ & $2 n^{2}-n$ \\
$M$ & 0 & 1 & 2 & $s-1$ & $n-1$ \\
$v M$ & 0 & $2 n$ & $4 n$ & $(s-1) 2 n$ & $2 n^{2}-2 n$ \\
$\rho$ & $2 n-1$ & $2 n-2$ & $2 n-3$ & $2 n-s$ & $n$ \\
\hline$S$ & $n-1 / 2$ & $n-1$ & $n-3 / 2$ & $n-s / 2$ & $n / 2$ \\
$\#$ & $n$ & $n-1$ & $n-2$ & $n-(s-1)$ & 1 \\
\hline
\end{tabular}

При $X=n$ достигается разность

$$
S(X)-\#(X)=\frac{n}{2}-1 .
$$

Она сколь угодно велика, если $n$ достаточно велико. 
ЗАмЕчАНиЕ. Причиной большого значения разности является большая величина $\left(a_{2}=2 n-1\right)$ второго неполного частного цепной дроби $k$.

Как мы сейчас увидим, это соображение позволяет построить такие иррациональные числа $k$, для которых значение параметра Колмогорова $\lambda_{n}$ арифметической прогрессии $\{k x, x=1,2, \ldots, n\}$,

$$
\lambda_{n}=\frac{\sup _{X}|S(X)-\#(X)|}{\sqrt{n}},
$$

не только не стремится к нулю при $n \rightarrow \infty$ (как это бывает для любого рационального значения $k$ ), но, наоборот, может принимать сколь угодно большие значения $\lambda_{n} \geqslant K$ бесконечное число раз (хотя и удовлетворяет неравенству $\left.\lambda_{n} \leqslant \sqrt{n}\right)$.

ТЕОрема 3. Предположим, что для цепной дроби

$$
k=\frac{1}{a_{1}+\frac{1}{a_{2}+\cdots}}
$$

неполное частное четного порядка, $a_{s+2}=2 r$, четно.

Тогда параметр Колмогорова арифметической прогрессии дробных долей $(\{k\},\{2 k\},\{3 k\}, \ldots)$ принимает для подходящей длиньь, $n=\left(q_{s}+q_{s+2}\right) / 2$, отрезка прогрессии значение $\lambda_{n}$, большее числа $K$, если неполное частное $a_{s+2}$ достаточно велико: для выполнения неравенства $\lambda_{n} \geqslant K$ достаточно, чтобы

$$
a_{s+2} \geqslant \alpha\left(a_{1}, \ldots, a_{s+1} ; K\right)
$$

где $\alpha$ - некоторая (явно выписанная) функция.

(Значение $\alpha$ явно указано в конце доказательства ниже, в неравенстве (3).)

ЗАмЕчАниЕ. Аналогичные оценки снизу получаются и для параметра стохастичности Мизеса, где вместо метрики равномерной сходимости используются средние квадратичные - величина $\alpha$ должна для этого быть лишь немного бо́льшей.

ЗАМЕчАНИЕ. Слишком большие значения параметра стохастичности $\lambda_{n}$ для данной последовательности делают гипотезу о случайности этой последовательности так же маловероятной, как и слишком малые.

ДокАЗАтЕЛьСтво. На плоскости с координатами $(x, m)$ рассмотрим векторы (рис. 5)

$$
\xi_{s}: \quad\left(x=q_{s}, m=p_{s}\right),
$$

соответствующие подходящим дробям $k \approx p_{s} / q_{s}$.

Если $s$ четно, то такой вектор $\xi_{s}$ лежит ниже прямой $m=k x$, а если нечетно то выше.

По теории цепных дробей, имеет место тождество

$$
\xi_{s+2}=\xi_{s}+a_{s+2} \xi_{s+1} .
$$




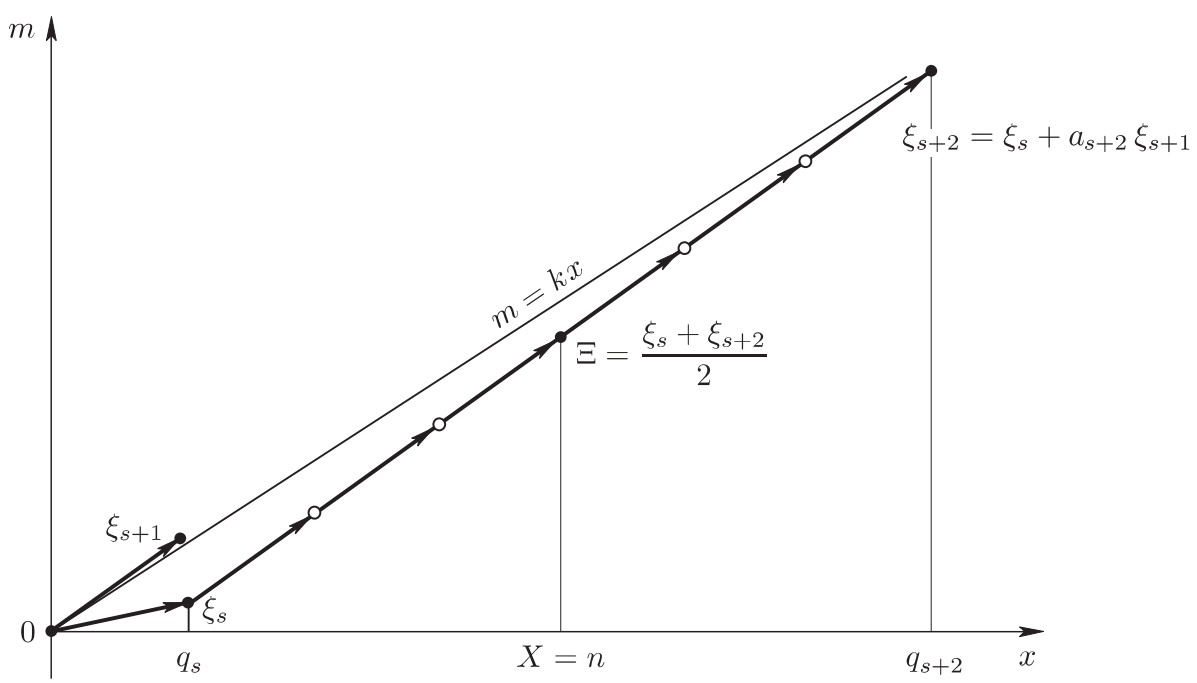

Рис. 5. Построение целочисленного вектора $\Xi$ (среднего между $\xi_{s}$ и $\left.\xi_{s+2}\right)$

Рассмотрим средний между $\xi_{s}$ и $\xi_{s+2}$ (оба индекса четны) целочисленный вектор (мы предполагаем четность числа $a_{s+2}=2 r$, чтобы получить целочисленный средний вектор)

$$
\Xi=\xi_{s}+r \xi_{s+1}=\frac{\xi_{s}+\xi_{s+2}}{2}
$$

с компонентами

$$
n=X=q_{s}+r q_{s+1}, \quad M=p_{s}+r p_{s+1} .
$$

Построим параллелограмм П, соответствующий этому выбору целых чисел

$$
n=X=\frac{q_{s}+q_{s+2}}{2}
$$

(см. рис. 6).

В этом параллелограмме нет никаких целых точек, кроме точки $\Xi$ (согласно теории цепных дробей), поэтому число целых точек в параллелограмме П составляет $\#(X)=1$ в нашем случае.

Оценим теперь снизу площадь параллелограмма П.

Эта площадь равна произведению высоты $n$ параллелограмма П на длину $R$ основания $A \Xi$ :

$$
S(X)=n R=n|A \Xi| .
$$

Заметим, что $A \Xi-$ средняя линия трапеции $C D \xi_{s+2} \xi_{s}$ :

$$
|A \Xi|=\frac{\left|C \xi_{s}\right|+\left|D \xi_{s+2}\right|}{2} \geqslant \frac{\left|C \xi_{s}\right|}{2} .
$$

Из подобия треугольников $\left(O C \xi_{s}\right)$ и $(O D E)$ находим

$$
\left|C \xi_{s}\right|=|D E| \frac{q_{s}}{q_{s+2}} \geqslant\left|\xi_{s+2} E\right| \frac{q_{s}}{q_{s+2}} .
$$




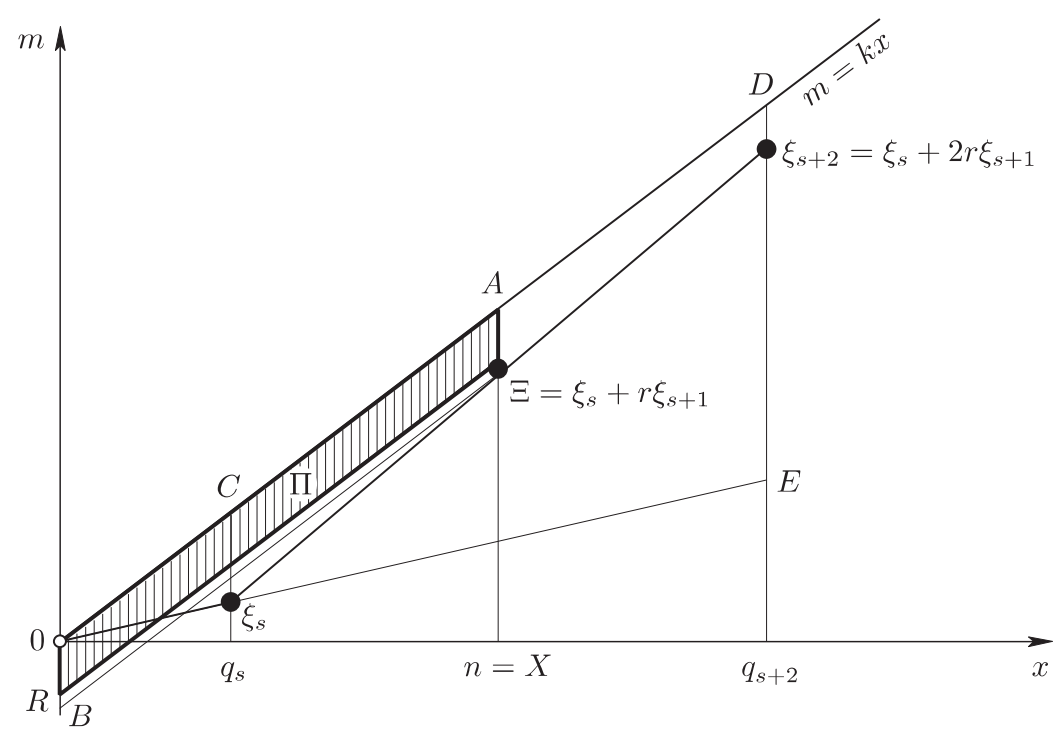

Рис. 6. Параллелограмм П большой (при большом $r$ ) площади, содержащий мало целых точек

Ордината точки $\xi_{s+2}$ есть $p_{s+2}$, а ордината точки $E$ есть $p_{s} q_{s+2} / q_{s}$. Поэтому произведение в правой части последнего неравенства равно следующей площади $[\cdot, \cdot]$ параллелограмма:

$$
q_{s}\left|\xi_{s+2} E\right|=\left|p_{s+2} q_{s}-p_{s} q_{s+2}\right|=\left|\left[\xi_{s+2}, \xi_{s}\right]\right| .
$$

Из тождества (2) мы находим эту площадь:

$$
\left[\xi_{s+2}, \xi_{s}\right]=a_{s+2}\left[\xi_{s+1}, \xi_{s}\right]
$$

так что, окончательно,

$$
q_{s}\left|\xi_{s+2} E\right|=a_{s+2}
$$

(поскольку натянутый на векторы $\xi_{s}$ и $\xi_{s+1}$ параллелограмм имеет площадь 1 , согласно теории цепных дробей).

Собирая доказанные неравенства, мы получаем для длины стороны параллелограмма П оценку снизу

$$
|A \Xi| \geqslant \frac{\left|C \xi_{s}\right|}{2} \geqslant \frac{q_{s}\left|\xi_{s+2} E\right|}{2 q_{s+2}}=\frac{a_{s+2}}{2 q_{s+2}} .
$$

Для площади этого параллелограмма получается поэтому такая оценка снизу:

$$
S(X)=n|A \Xi| \geqslant \frac{q_{s}+q_{s+2}}{2} \frac{a_{s+2}}{2 q_{s+2}} \geqslant \frac{a_{s+2}}{4} .
$$

Теорема 3 легко выводится из этого неравенства, но я приведу и явную оценку снизу $\alpha$ величины $a_{s+2}$. 
Если $S \geqslant 2$, то $S-1 \geqslant S / 2$, поэтому для параллелограмма П выполняется неравенство

$$
|S(X)-\#(X)|=S(X)-1 \geqslant \frac{S}{2} \geqslant \frac{a_{s+2}}{8} .
$$

Для параметра Колмогорова

$$
\lambda_{n}=\frac{\max _{X}|S(X)-\#(X)|}{\sqrt{n}}
$$

мы получаем отсюда оценку снизу:

$$
\lambda_{n} \geqslant K
$$

если $a_{s+2} / 8 \geqslant K \sqrt{n}$. Для этого достаточно выполнение неравенства $a_{s+2}^{2} \geqslant$ $64 K^{2} n$, т. е. неравенства

$$
a_{s+2}^{2} \geqslant 32 K^{2}\left(q_{s}+q_{s+2}\right) .
$$

Поскольку по формуле (2) имеем

$$
q_{s+2}=q_{s}+a_{s+2} q_{s+1} \leqslant\left(1+a_{s+2}\right) q_{s+1},
$$

то мы заключаем, что для выполнения неравенства $\lambda_{n} \geqslant K$ достаточно выполнение неравенства

$$
a_{s+2}^{2} \geqslant 32 K^{2}\left(2+a_{s+2}\right) q_{s+1},
$$

которое можно переписать в виде

$$
\left(a_{s+2}-16 K^{2} q_{s+1}\right)^{2} \geqslant 256 K^{4} q_{s+1}^{2}+64 K^{2} q_{s+1} .
$$

Это последнее неравенство выполняется при условии

$$
a_{s+2} \geqslant 16 K^{2} q_{s+1}+8 K \sqrt{4 K^{2} q_{s+1}^{2}+q_{s+1}}
$$

что и доказывает теорему 3 (со значением $\alpha$, указанным в правой части неравенства (3)).

ЗАмЕчАниЕ. Я не знаю, будет ли значение параметра случайности Колмогорова $\lambda_{n}$ арифметической прогрессии дробных долей $n$ чисел $k x$ стремиться к нулю для почти всех (в смысле меры Лебега) действительных чисел $k$, или будет ли он так же часто стремиться к бесконечности, или будет ли он, как правило, отделен от 0 и от $\infty)$. Эргодичность системы Гаусса-Кузьмина, $z \mapsto\{1 / z\}$, подсказывает, что любое определенное таким образом предельное поведение параметра $\lambda_{n}$ должно иметь вероятность либо 0, либо 1 (если оно зависит только от асимптотики элементов $a_{s}$ цепной дроби при $\left.s \rightarrow \infty\right)$. Но будет ли вероятность того или иного ответа на приведенные выше вопросы о типичности разных видов поведения равна 0 или 1, я не знаю. 


\section{Список литературы}

[1] A. Kolmogoroff, "Sulla determinazione empirica di una legge di distribuzione", Giorn. Ist. Ital. Attuari, 4:1 (1933), 83-91 (Italian); pyc. пер.: А. Н. Колмогоров, "Об эмпирическом определении закона распределения", [Избр. труды], Теория вероятностей и математическая статистика, Наука, М., 1986, 134-141; англ. пер.: A. N. Kolmogorov, "On the empirical determination of a distribution law", Selected works. Vol. II. Probability theory and mathematical statistics, Math. Appl. (Soviet Ser.), 26, Kluwer Acad. Publ., Dordrecht, 1992, 139-146.

[2] V.I. Arnold, "Empirical study of stochasticity for deterministic classical dynamics of geometrical progressions of residues", Funct. Anal. Other Math., 2:4 (2007) (to appear).

[3] В.И. Арнольд, Динамика, статистика и проективная геометрия полей Галуа (лекция 13.11.2004), МЦНМО, Москва, 2005; англ. пер.: V. I. Arnol'd, Dynamics, statistics and projective geometry of Galois fields (lecture on 13 November 2004), MCCME, Moscow, 2005.

[4] А.Н. Колмогоров, "Об одном новом подтверждении законов Менделя", Докл. АН CCCP, 27:1 (1940), 37-41; англ. пер.: A. N. Kolmogorov, "On a new confirmation of Mendel's laws", Selected works. Vol. II. Probability theory and mathematical statistics, Math. Appl. (Soviet Ser.), 26, Kluwer Acad. Publ., Dordrecht, 1992, 222-227.

[5] В. И. Арнольд, "Слабые асимптотики чисел решений диофантовых задач", Функи. анализ и его прил., 33:4 (1999), 65-66; англ. пер.: V. I. Arnol'd, "Weak asymptotics for the numbers of solutions of Diophantine problems", Funct. Anal. Appl., 33:4 (1999), $292-293$.

В. И. Арнольд (V. I. Arnold)

Поступила в редакцию

Математический институт

10.12.2007

им. В. А. Стеклова РАН 